\title{
A Kähler Compatible Moyal Deformation of the First Heavenly Equation
}

\author{
Marco MACEDA and Daniel MARTÍNEZ-CARBAJAL \\ Departamento de Física, Universidad Autónoma Metropolitana, Av. San Rafael Atlixco 186, \\ C.P. 03340, Deleg. Iztapalapa, Mexico City, México \\ E-mail:mmac@xanum.uam.mx,danielmc@xanum.uam.mx
}

Received June 07, 2019, in final form September 08, 2019; Published online September 22, 2019

https://doi.org/10.3842/SIGMA.2019.073

\begin{abstract}
We construct a noncommutative Kähler manifold based on a non-linear perturbations of Moyal integrable deformations of $D=4$ self-dual gravity. The deformed Kähler manifold preserves all the properties of the commutative one, and we obtain the associated noncommutative Kähler potential using the Moyal deformed gravity approach. We apply this construction to the Atiyah-Hitchin metric and its Kähler potential, which is useful in the description of interactions among magnetic monopoles at low energies.
\end{abstract}

Key words: heavenly equations; Moyal deformation; Atiyah-Hitchin metric

2010 Mathematics Subject Classification: 37K10; 53C26; 53D55; 70H06; 83C20

\section{Introduction}

Several applications of hyper-Kähler manifolds in four dimensions involve gravitational instantons, non-linear graviton theory and the heavenly equations [12, 17, 25, 31, 32]; they have also been extensively used in supersymmetric field theories [2, 22]. Of particular interest is their appearance in topological field theories and string theory, where in some cases the moduli spaces have a hyper-Kähler structure. The existence of such a structure provides a more profound and alternative understanding of a physical system in general.

Even though hyper-Kähler manifolds have been analysed in great detail, there exist examples where the metric has proved to be difficult or impossible to calculate. Nevertheless, an algebraic description of four-dimensional non-compact hyper-Kähler manifolds possessing one abelian isometry was given in [7]. In that work, a self-duality condition for the Killing vector associated to the isometry plays a fundamental role in the analysis and classification manifolds; the translational or rotational character of the isometry translates into the existence of a translational or rotational Killing vector.

Two particular examples of self-dual manifolds with rotational Killing symmetries are the Eguchi-Hanson and the Taub-NUT metrics; both metrics share $\mathrm{SO}(3) \times \mathrm{SO}(2)$ as a larger group of isometries, but these two group factors act differently on each spacetime. $\mathrm{SO}(3)$ is a translation symmetry for the Eguchi-Hanson metric with $\mathrm{SO}(2)$ acting as a rotational one; the situation is the opposite for the Taub-NUT spacetime. On the other hand, a relevant example of a spacetime admitting only a rotational isometry is the Atiyah-Hitchin $(\mathrm{AH})$ metric that arises on the moduli space $M_{2}^{0}$ of BPS SU(2) monopoles [4, 18].

As noted in a series of works $[3,4,18]$, the AH spacetime is a useful tool in the description of interactions among magnetic monopoles. More specifically, the $\mathrm{AH}$ metric is the metric on the moduli space of charge-two non-abelian magnetic $\mathrm{SU}(2)$ monopole with a fixed centre. Its geodesics describe low-energy monopoles interacting through the exchange of massless photons and scalars [4]; at long distances, it reduces to the Taub-NUT space with negative mass parameter [21]. The structure of the AH metric is a four-dimensional hyper-Kähler manifold 
with $\mathrm{SO}(3)$ isometry; the $\mathrm{SO}(3)$ group does not rotate the three Kähler forms, and it is the only specific example of a four-dimensional hyper-Kähler space without tri-holomorphic isometries. Furthermore, the AH spacetime is a self-dual solution to Einstein field equations [5] and all of its Killing vectors lack a self-dual covariant derivative [20]; the isometry group $\mathrm{SU}(2)$ is often identified with a supersymmetry group [19].

In general, the metric related to self-dual vacuum solutions to Einstein's field equations is a solution to the first, or the second, heavenly equations [34]. The original motivation for heavenly equations, heavenly metrics and heavenly spaces was the desire to obtain real solutions to Einstein's equations on real manifolds and spawned several papers on the subject and its generalisations $[1,8,13,14,23]$. Assuming that the anti-self-dual part of the Weyl tensor was algebraically special, the equivalence between the vacuum Einstein field equations in complex spacetime and the heavenly equation was established in [36]. The heavenly equations are also integrable using the twistor formalism [10,31] and several examples are known [15, 37]; they are a constant source of research in mathematics and physics.

It is of interest to consider integrable generalisations of the heavenly equation due to its applications to physical systems; its modifications may allow a description of new phenomena or interactions not present in the standard description of a system. For instance, one of the possible generalisations that may be relevant is the one related to the Moyal $\star$-product. Within the context of particles and fields, the Moyal product provides a straightforward generalisation to noncommutative field theories and introduces, for example, new interactions in the Standard Model that may be probed in the laboratory. Several applications in quantum gravity also exist, in particular regarding the issue of the singularities and the thermodynamical properties of solutions in general relativity.

In the case of the first heavenly equation, a Moyal deformation of this equation has been done independently by Strachan [38] and Takasaki [43]. Furthermore, a suitable deformed differential calculus was introduced in [41] to deal with the deformation. When applied to integrable systems, the equations

$$
\mathrm{d} \widehat{\boldsymbol{\Omega}}=0, \quad \widehat{\boldsymbol{\Omega}} \wedge \widehat{\boldsymbol{\Omega}}=0,
$$

for a 2 -form $\widehat{\boldsymbol{\Omega}}$ provide a concise writing of the integrability conditions for the deformed system.

We want to analyse the consequences of a noncommutative structure on the moduli space of interacting magnetic monopoles; we expect that such a deformation gives rise to interactions that may be identified with some already known or produces new ones. For the classical case, the Kähler potential for the AH metric was obtained by Olivier [28] following an approach based on the existence of a $\eta$-self-dual Killing vector in conjunction with previous results obtained by Boyer and Finley [9]. In our approach, we consider a Moyal deformation of this Kähler potential, and we require that the deformed potential $\widehat{\Omega}$ must share the same features and properties of the classical one.

The plan of the present paper is as follows. In Section 2, we review the noncommutative deformation of the Monge-Ampère or first heavenly equation using the Moyal $\star$-product; in this section, we write the equation satisfied by the noncommutative contributions that preserve the first heavenly equation. In Section 3, we also recall the anti-self-dual vacuum Einstein equations that determine the structure of complex four-dimensional metrics of Euclidean signature. How integrability is preserved in the Moyal-deformed case for these spaces and the conditions under which we guarantee that the Moyal-deformed potential is Kähler are presented there.

Afterwards, in Section 4, we use the Moyal deformed gravity approach to rewrite the deformed Kähler potential $\widehat{\Omega}$ in terms of local frame fields $\hat{e}_{\mu}^{a}(x, \theta)$; we also obtain general expressions for the corresponding deformed metric elements. We discuss the deformed Kähler potential up to first order on the noncommutative parameter $\theta$ in greater detail in Section 5; through an integration procedure, we explicitly specify the first order modifications to the deformed metric 
and vierbien. The results in Sections 4 and 5 are put together in Section 6 to analyse the case of the AH metric. There, we obtain the Moyal deformed AH metric as a function of the original AH metric, the corrections to the Kähler manifold and the complex coordinates used in the original formulation by Olivier. We finally end with our conclusions and some remarks on future work.

\section{Moyal deformation of the first heavenly equation}

It is well known that the first and second heavenly equations describe the general metric of a selfdual vacuum space-time [34] and that these equations are integrable by twistor methods [10, 31, 33]. Plebański [34] showed that complex metrics with a self-dual Riemann tensor, to be referred to as self-dual metrics, can be described in terms of one function $\Omega$, the Kähler potential, satisfying the first heavenly equation

$$
\left\{\Omega_{p}, \Omega_{q}\right\}_{\mathrm{PB}}=\Omega_{p \bar{p}} \Omega_{q \bar{q}}-\Omega_{p \bar{q}} \Omega_{q \bar{p}}=1 .
$$

This equation is also called Plebański's first equation; it defines a completely multidimensional integrable system with an infinite number of conservation laws, hierarchy and Lax pair formulation $[27,29,30,39,40]$. The Kähler potential $\Omega$ is an unknown function of suitable spacetime coordinates $x^{\mu}:=(p, q, \bar{p}, \bar{q})$; it gives a local expression of self-dual vacuum Einstein spaces.

In this section we outline the procedure in [38] to obtain the deformation of the first heavenly equation using Strachan's idea. The starting point is to replace the Poisson bracket

$$
\{F, G\}_{\mathrm{PB}}=\frac{\partial F}{\partial \bar{p}} \frac{\partial G}{\partial \bar{q}}-\frac{\partial F}{\partial \bar{q}} \frac{\partial G}{\partial \bar{p}},
$$

by the Moyal bracket defined as [26]

$$
\{F, G\}_{\mathrm{MB}}:=\frac{1}{\mathrm{i} \theta}(F \star G-G \star F)=\frac{2}{\theta} F \sin \left[\frac{\theta}{2}\left(\frac{\overleftarrow{\partial}}{\partial \bar{p}} \frac{\vec{\partial}}{\partial \bar{q}}-\frac{\overleftarrow{\partial}}{\partial \bar{q}} \frac{\vec{\partial}}{\partial \bar{p}}\right)\right] G
$$

with the $\star$-product defined as

$$
f \star g:=f \exp \left[\frac{\mathrm{i} \theta}{2}\left(\frac{\overleftarrow{\partial}}{\partial \bar{p}} \frac{\vec{\partial}}{\partial \bar{q}}-\frac{\overleftarrow{\partial}}{\partial \bar{q}} \frac{\vec{\partial}}{\partial \bar{p}}\right)\right] g
$$

Thus the Moyal algebra is a deformation of the Poisson algebra. Using the well-known Taylor expansion of the sine function we obtain

$$
\{F, G\}_{\mathrm{MB}}=\sum_{s}^{\infty}-2 \mathrm{i} \theta^{2 s}\left(F *^{2 s+1} G\right),
$$

where the product $*^{2 s+1}$ has the generic form

$$
f(x) *^{r} g(x)=\frac{1}{r !}\left(\frac{\mathrm{i}}{2}\right)^{r} \varepsilon^{\mu_{1} \nu_{1}} \cdots \varepsilon^{\mu_{r} \nu_{r}} \partial_{\mu_{1}} \cdots \partial_{\mu_{r}} f(x) \partial_{\nu_{1}} \cdots \partial_{\nu_{r}} g(x) .
$$

The above formulation can be also implemented by considering a noncommutative matrix

$$
\theta^{\mu \nu}:=\theta \varepsilon^{\mu \nu}=\theta \delta_{\bar{\imath}}^{\mu} \delta_{\bar{\jmath}}^{\nu} \epsilon^{\bar{\jmath} \bar{\jmath}}=\theta\left(\begin{array}{cccc}
0 & 0 & 0 & 0 \\
0 & 0 & 0 & 0 \\
0 & 0 & 0 & 1 \\
0 & 0 & -1 & 0
\end{array}\right) .
$$


with the $\star$-product defined as

$$
f \star g:=f \exp \left(\frac{\mathrm{i}}{2} \theta^{\mu \nu} \frac{\overleftarrow{\partial}}{\partial x^{\mu}} \frac{\vec{\partial}}{\partial x^{\nu}}\right) g
$$

In the above, $\varepsilon^{\mu \nu}=-\varepsilon^{\nu \mu}$ is an anti-symmetric object, and $\epsilon^{\bar{i} \bar{\jmath}}$ is the standard Levi-Civita tensor in two dimensions. Following [38] and [35], we briefly review the iterative method for constructing a set of differential equations for the Moyal deformation of the first heavenly equation. For this purpose, we consider then a series development in powers of $\theta$ for $\widehat{\Omega}$

$$
\widehat{\Omega}=\sum_{n=0}^{\infty} \theta^{n} \Omega^{(n)}, \quad n=0,1, \ldots
$$

Here $\Omega^{(n)}$ are functions to be determined and $\Omega^{(0)}=: \Omega$ is the classical Kähler potential. Plugging this expansion into the integrable deformation of Plebański's (first heavenly) equation

$$
\left\{\widehat{\Omega}_{p}, \widehat{\Omega}_{q}\right\}_{\mathrm{MB}}=1,
$$

we obtain the expression

$$
\left\{\widehat{\Omega}_{p}, \widehat{\Omega}_{q}\right\}_{\mathrm{MB}}=-2 \mathrm{i} \sum_{r=0}^{\infty} \theta^{r} \sum_{s=0}^{\left[\frac{r}{2}\right]} \sum_{m=0}^{r-2 s}\left(\partial_{p} \Omega^{(m)} *^{2 s+1} \partial_{q} \Omega^{(r-m-2 s)}\right)=1 .
$$

We compare now the coefficients of the same powers of $\theta$ in both sides of this equation; for $r=0$ we find the first heavenly equation (2.1). For any $r \geq 1$, equation (2.5) gives the condition

$$
\sum_{s=0}^{\left[\frac{r}{2}\right]} \sum_{m=0}^{r-2 s}\left(\partial_{p} \Omega^{(m)} *^{2 s+1} \partial_{q} \Omega^{(r-m-2 s)}\right)=0 .
$$

To analyse the lowest order modifications to the first heavenly equation, we set $r=1$ to obtain

$$
\Omega_{p \bar{p}} \Omega_{q \bar{q}}^{(1)}+\Omega_{p \bar{p}}^{(1)} \Omega_{q \bar{q}}-\Omega_{p \bar{q}} \Omega_{q \bar{p}}^{(1)}-\Omega_{p \bar{q}}^{(1)} \Omega_{q \bar{p}}=0 .
$$

Therefore, once $\Omega$ is known, equation (2.7) becomes a linear partial differential equation for the first order corrections $\Omega^{(1)}$. The deformed potential $\widehat{\Omega}$ defined as before is not necessarily Kählerlike, we need to impose additional conditions to guarantee that it will be; these conditions will be discussed in the next section.

\section{$3 \quad$ Integrable systems}

Several multidimensional integrable systems were discussed in [41] assuming a symplectic manifold with some associated $\star$-product. All these systems share the characteristic feature that they have associated a 2 -form $\widehat{\Omega}$ which satisfies the equations

$$
\begin{aligned}
& \mathrm{d} \widehat{\boldsymbol{\Omega}}=0, \\
& \widehat{\boldsymbol{\Omega}} \wedge \widehat{\boldsymbol{\Omega}}=0 .
\end{aligned}
$$

These equations contain the integrability conditions of the systems in a concise geometric way. In the following, we focus on the implications of these relations for the particular case of an integrable deformation of self-dual vacuum Einstein equations. 


\subsection{The self-dual vacuum Einstein equations}

Consider a real manifold $(\mathcal{M}, g)$ of dimension four and metric

$$
\mathrm{d} s^{2}=g_{a b} \mathrm{~d} \phi^{a} \mathrm{~d} \phi^{b}, \quad a=1, \ldots, 4 .
$$

We assume that the associated Levi-Civita covariant derivative is torsionless, i.e., $D_{k} g_{a b}=0$. Locally it is possible to introduce complex coordinates $\phi^{a}=\left(\tau^{i}, \tau^{\bar{\imath}}\right), i, \bar{\imath}=1,2$, such that the metric reads

$$
\mathrm{d} s^{2}=g_{i \bar{\jmath}} \mathrm{d} \tau^{i} \mathrm{~d} \tau^{\bar{\jmath}}+g_{\bar{\imath} j} \mathrm{~d} \tau^{\bar{\imath}} \mathrm{d} \tau^{j}=2 g_{i \bar{\jmath}} \mathrm{d} \tau^{i} \mathrm{~d} \tau^{\bar{\jmath}} .
$$

Note that this metric is real since it was so in the original coordinates; as a consequence we have

$$
g_{i \bar{\jmath}}=g_{j \bar{\imath}}^{*}, \quad g_{i j}=g_{\bar{\jmath}}^{*}=0 .
$$

The indexes $i$ and $\bar{\imath}$ are called holomorphic and antiholomorphic respectively; the standard convention is to write the holomorphic index first.

Complex 4-metrics of Euclidean signature with vanishing Ricci tensor and anti-self-dual Weyl tensor correspond to anti-self-dual vacuum Einstein solutions. Using the fact that these metrics are Kähler, they may be written in terms of the Kähler potential $\Omega$ as $g_{i \bar{\jmath}}:=\Omega_{i \bar{\jmath}}:=\partial_{i \bar{\jmath}} \Omega$. It follows that

$$
g_{\mu \nu}=\left(\delta_{\mu}^{i} \delta_{\nu}^{\bar{\jmath}}+\delta_{\nu}^{i} \delta_{\mu}^{\bar{\jmath}}\right) \Omega_{i \bar{\jmath}}=\left(\begin{array}{cc}
0 & \Omega_{i \bar{\jmath}} \\
\Omega_{j \bar{\imath}} & 0
\end{array}\right) .
$$

As mentioned before, a noncommutative deformation of the integrability conditions equations (3.1) and (3.2) was proposed in [41]. More specifically, it was noted that if $\widehat{\boldsymbol{\Omega}}$ is the deformed 2-form

$$
\widehat{\boldsymbol{\Omega}}=\mathrm{d} p \wedge \mathrm{d} q+\lambda\left(\widehat{\Omega}_{p \bar{p}} \mathrm{~d} p \wedge \mathrm{d} \bar{p}+\widehat{\Omega}_{p \bar{q}} \mathrm{~d} p \wedge \mathrm{d} \bar{q}+\widehat{\Omega}_{q \bar{p}} \mathrm{~d} q \wedge \mathrm{d} \bar{p}+\widehat{\Omega}_{q \bar{q}} \mathrm{~d} q \wedge \mathrm{d} \bar{q}\right)+\lambda^{2} \mathrm{~d} \bar{p} \wedge \mathrm{d} \bar{q},
$$

then it clearly satisfies the condition $\mathrm{d} \widehat{\boldsymbol{\Omega}}=0$. Furthermore, it is straightforward to see that

$$
\widehat{\boldsymbol{\Omega}} \wedge \widehat{\boldsymbol{\Omega}}=\lambda^{2}\left(\left\{\widehat{\Omega}_{p}, \widehat{\Omega}_{q}\right\}_{\mathrm{MB}}-1\right) \mathrm{d} p \wedge \mathrm{d} \bar{p} \wedge \mathrm{d} q \wedge \mathrm{d} \bar{q} .
$$

The right hand side of this equation vanishes if $\widehat{\Omega}$ satisfies the deformed Plebański equation. This result means that a Moyal deformation of the first heavenly equation preserves its integrability. Furthermore, we have the important result that a perturbative solution equation (2.4) exists. This result implies that equation (3.4) generalises to

$$
g_{\mu \nu}^{(n)}=\left(\delta_{\mu}^{i} \delta_{\nu}^{\bar{\jmath}}+\delta_{\nu}^{i} \delta_{\mu}^{\bar{\jmath}}\right) \Omega_{i \bar{\jmath}}^{(n)} .
$$

\subsection{Deformed properties}

As we previously mentioned, we want to construct a four-dimensional Moyal deformed integrable Kähler manifold. For this purpose, we impose that the deformed Kähler potential $\widehat{\Omega}$ must share the same features and properties of the undeformed system; they are $\left(\hat{g}_{i \bar{\jmath}}:=\partial_{i} \partial_{\bar{\jmath}} \widehat{\Omega}\right)$

1) the 2 -form $\widehat{\boldsymbol{\Omega}}:=\widehat{\Omega}_{i \bar{\jmath}} \mathrm{d} x^{i} \wedge \mathrm{d} \bar{x}^{\bar{\jmath}}:=2 \mathrm{i} \hat{g}_{i \bar{\jmath}} \mathrm{d} x^{i} \wedge \mathrm{d} \bar{x}^{\bar{\jmath}}$ should be closed, i.e., $\mathrm{d} \widehat{\boldsymbol{\Omega}}=0$,

2) the metric coefficients $\widehat{\Omega}_{i \bar{j}}$ should be real (hermitian property),

3) the determinant of $\widehat{\Omega}_{i \bar{\jmath}}$ should be equal to one, i.e., $\operatorname{det} \widehat{\Omega}_{i \bar{\jmath}}:=\widehat{\Omega}_{p \bar{p}} \star \widehat{\Omega}_{q \bar{q}}-\widehat{\Omega}_{p \bar{q}} \star \widehat{\Omega}_{q \bar{p}}=1$. 
The first of the above conditions can be analysed by fixing for the 2 -form $\widehat{\boldsymbol{\Omega}}$, the same functional form as that of equation (3.5). This fact implies that the following condition must hold

$$
\partial_{k} \Omega_{i \bar{\jmath}}^{(n)}=0=\partial_{\bar{k}} \Omega_{i \bar{\jmath}}^{(n)}, \quad k=p, q, \quad \bar{k}=\bar{p}, \bar{q} .
$$

We now write equation (2.6) as

$$
\sum_{m=0}^{r}\left(\partial_{p} \Omega^{(m)} *^{1} \partial_{q} \Omega^{(r-m)}\right)+\sum_{s=1}^{\left[\frac{r}{2}\right]} \sum_{m=0}^{r-2 s}\left(\partial_{p} \Omega^{(m)} *^{2 s+1} \partial_{q} \Omega^{(r-m-2 s)}\right)=0 .
$$

The second sum vanishes because of the first property imposed on $\Omega^{(n)}$; we have thus

$$
\sum_{m=0}^{r}\left(\partial_{p} \Omega^{(m)} *^{1} \partial_{q} \Omega^{(r-m)}\right)=\sum_{m=0}^{r} \epsilon^{\overline{\bar{\jmath}}} \Omega_{p \bar{\imath}}^{(m)} \Omega_{q \bar{\jmath}}^{(r-m)}=0 .
$$

Therefore, we can write equation (2.5) in terms of the Poisson bracket as

$$
\left\{\widehat{\Omega}_{p}, \widehat{\Omega}_{q}\right\}_{\mathrm{PB}}=1 \text {. }
$$

The second condition implies that the corresponding metric coefficients are hermitian as in the commutative case; therefore, each perturbation $\Omega^{(n)}$ is also hermitian. We have then

$$
\Omega_{i \bar{\jmath}}^{(n)}=\Omega_{j \bar{\imath}}^{\dagger(n)}, \quad \Omega_{i j}^{(n)}=\Omega_{\bar{\imath} \bar{\jmath}}^{\dagger(n)}=0,
$$

where the last two conditions hold because we have a deformed Kähler manifold.

The third property can be imposed from the curvature condition equation (2.1) since the deformed first heavenly equation admits a rewriting as a simple determinant. We know that in the case of the non-deformed Kähler manifold, the determinant of the metric tensor is equal to one. In the case of the deformed case the deformed metric tensor $\widehat{\Omega}_{i \bar{\jmath}}$ should then satisfy the same property. We demand that by definition

$$
\operatorname{det} \widehat{\Omega}_{i \bar{\jmath}}:=\widehat{\Omega}_{p \bar{p}} \widehat{\Omega}_{q \bar{q}}-\widehat{\Omega}_{p \bar{q}} \widehat{\Omega}_{q \bar{p}}=1 .
$$

The above equation is equivalent to $\operatorname{det} \widehat{\Omega}_{i \bar{\jmath}}=\epsilon^{\bar{k} l} \widehat{\Omega}_{p \bar{k}} \widehat{\Omega}_{q \bar{l}}=1$. Using the power series expansion in equation (2.4) for $\widehat{\Omega}$, we get the different contributions to $\operatorname{det} \widehat{\Omega}_{i \bar{\jmath}}$ order by order on $\theta$

$$
\operatorname{det} \widehat{\Omega}_{i \bar{\jmath}}=\left\{\Omega_{p}, \Omega_{q}\right\}_{\mathrm{PB}}+\sum_{r=0}^{\infty} \sum_{\substack{m, n=0 \\ m+n=r \geq 1}}^{r} \epsilon^{\bar{k} \bar{l}} \theta^{m+n} \Omega_{p \bar{k}}^{(m)} \Omega_{q \bar{l}}^{(n)} .
$$

The first term in this expression is the Moyal deformation of the first heavenly equation; its value is equal to one. Therefore, we conclude that the second term should vanish, namely

$$
\sum_{\substack{m, n=0 \\ m+n=r \geq 1}}^{r} \epsilon^{\bar{k} \bar{l}} \theta^{m+n} \Omega_{p \bar{k}}^{(m)} \Omega_{q \bar{l}}^{(n)}=0
$$

In this equation are encoded all the combinations of order $\theta^{j}$ such that $j>r$.

\section{Noncommutative gravity}

In the previous section, we studied the noncommutative deformation of the Kähler potential using the Moyal deformation of the first heavenly equation. The deformation functions $\Omega^{(n)}$ are unknown; each one of them satisfy their respective differential equations obtained from equation (2.6). In this section, we give an ansatz for the deformed Kähler potentials $\Omega^{(n)}$ appearing in equation (3.7). As we will see later, the deformed functions $\Omega^{(n)}$ can be expressed in terms of a deformed local frame or vierbein $\hat{e}_{\mu}^{a}(x, \theta)$; after an integration procedure, the deformed Kähler potential $\widehat{\Omega}$ will be written in terms of the vierbein. 


\subsection{Deformed gauge fields}

For some time now, a subject of interest has been the construction of consistent noncommutative deformations of Einstein gravity. Following the standard procedure to construct noncommutative gauge and scalar field theories [11, 42], noncommutative versions of the Einstein-Hilbert action have been obtained by replacing the ordinary product by the noncommutative Moyal product in equation (2.3). The noncommutative structure of spacetime is then

$$
\left[x^{\mu}, x^{\nu}\right]_{\star}=\mathrm{i} \theta^{\mu \nu},
$$

where the elements $\theta^{\mu \nu}$ are constant (canonical) parameters and antisymmetric, i.e., $\theta^{\mu \nu}=-\theta^{\nu \mu}$.

In this approach, we can introduce a noncommutative metric as

$$
\hat{g}_{\mu \nu}=\frac{1}{2}\left(\hat{e}_{\mu}^{a} \star \hat{e}_{\nu}^{b}+\hat{e}_{\nu}^{a} \star \hat{e}_{\mu}^{b}\right) \eta_{a b}
$$

in terms of a vierbein $\hat{e}_{\mu}^{a}(x, \theta)$ and the Minkowski metric $\eta_{a b}$; the vierbein $\hat{e}_{\mu}^{a}(x, \theta)$ reduces to the commutative one when $\theta=0$. The metric $\hat{g}_{\mu \nu}$ is symmetric by construction and real even if the deformed tetrad fields $\hat{e}_{\mu}^{a}(x, \theta)$ are complex quantities. For $\theta=0$, we identify this metric with the commutative metric field

$$
\left.\hat{g}_{\mu \nu}(x, \theta)\right|_{\theta=0}=g_{\mu \nu}=e_{\mu}^{a} e_{\nu}^{b} \eta_{a b} .
$$

We want now to construct a Moyal deformed spacetime with associated deformed vierbein and metric, sharing the same properties of the undeformed spacetime, and such that the deformed Kähler potential discussed in the Section 3.2 exists. For this purpose, we first introduce the vector fields $\hat{e}_{\mu}^{b}$ as

$$
\hat{e}_{\mu}^{b}=e_{\mu}^{b}+\theta^{k \lambda} e_{\mu k \lambda}^{b}+\cdots+\theta^{k_{1} \lambda_{1}} \cdots \theta^{k_{n} \lambda_{n}} e_{\mu k_{1} \lambda_{1} \cdots k_{n} \lambda_{n}}^{b}+\cdots
$$

where the elements $e_{\mu k_{1} \lambda_{1} \cdots k_{n} \lambda_{n}}^{b}$ are to be found. With this series expansion in powers of $\theta$, we write then the metric tensor as

$$
\hat{g}_{\mu \nu}=g_{\mu \nu}+\mathrm{i} \theta^{k \lambda} g_{\mu \nu k \lambda}^{(1)}+\mathcal{O}\left(\theta^{2}\right),
$$

up to first order on $\theta$.

Since we are interested in making compatible the noncommutative deformation of the Kähler metric equation (3.6), with the deformation in equation (4.2), let us assume the following decomposition for the verbein

$$
\theta^{k \lambda} e_{\mu k \lambda}^{b}=\theta^{k \lambda} \mathcal{P}_{k \lambda} e_{\mu}^{(1) b},
$$

to first order on $\theta$; here $\mathcal{P}_{k \lambda}$ and $e_{\mu}^{(1) b}$ are unknown quantities. For the $n$-th order we generalize this ansatz to

$$
\theta^{k_{1} \lambda_{1}} \cdots \theta^{k_{n} \lambda_{n}} e_{\mu k_{1} \lambda_{1} \cdots k_{n} \lambda_{n}}^{b}=\left(\theta^{k \lambda} \mathcal{P}_{k \lambda}\right)^{n} e_{\mu}^{(n) b}
$$

We now impose the condition

$$
\hat{e}_{\mu}^{b}=\sum_{n=0}^{\infty} \theta^{n} e_{\mu}^{(n) b}, \quad e_{\mu}^{(0) b}:=e_{\mu}^{b},
$$

implying $\theta^{k \lambda} \mathcal{P}_{k \lambda}=\theta$; it is easy to show now that if we choose $\mathcal{P}_{k \lambda}:=\partial_{k} \Omega_{p} \partial_{\lambda} \Omega_{q}$ where $\Omega$ is the undeformed Kähler potential, then equation (4.3) is satisfied, where $\theta^{k \lambda}$ is given by 
equation (2.2). In a similar way as for the vierbein, the tensor metric equation (4.2) takes the general form

$$
\hat{g}_{\mu \nu}=\sum_{n=0}^{\infty} \theta^{n} g_{\mu \nu}^{(n)},
$$

where the $g_{\mu \nu}^{(n)}$ 's are tensor fields written in terms of the $e_{\mu}^{(n) a}$, s; we fix their form as follows: first, to make compatible this deformation with the structure of a Kähler manifold, we need to impose the constraint $\partial_{\alpha} g_{\mu \nu}^{(n)}=0$ with $x^{\alpha}=(p, q, \bar{p}, \bar{q})$. This condition implies the property $\partial_{\mu} e_{\nu}^{(n) a}=0$ for the vierbein; equation (4.1) simplifies then to

$$
\hat{g}_{\mu \nu}=\hat{e}_{\mu}^{a} \hat{e}_{\nu}^{b} \eta_{a b}
$$

Using now the expansion of $\hat{e}_{\mu}^{a}$ in powers of $\theta$ into equation (4.4), and equating the coefficients of the same power of $\theta$ in both sides of the equation, we obtain the $n$-th tensor field $g_{\mu \nu}^{(n)}$ in terms of the deformed tetrad $e_{\mu}^{(n) a}$ as

$$
g_{\mu \nu}^{(n)}=\sum_{m=0}^{n} e_{\mu}^{(m) a} e_{\nu}^{(n-m) b} \eta_{a b}, \quad n=0,1, \ldots
$$

\section{The deformed Kähler potential}

According with [34], self-dual gravity can be parametrised in terms of the complex coordinates $x^{\mu}=\{p, q, \bar{p}, \bar{q}\}$ and the resulting spacetime has the estructure of a Kähler manifold. In our case, the Kähler spacetime given in equation (3.3) can be written in terms of the classical vierbein $e_{\mu}^{a}$ and the local flat spacetime metric $\eta_{a b}$ defined as

$$
e_{\mu}^{a}:=\left(\begin{array}{cccc}
0 & 0 & \Omega_{p \bar{p}} & \Omega_{p \bar{q}} \\
1 & 0 & 0 & 0 \\
0 & 0 & \Omega_{q \bar{p}} & \Omega_{q \bar{q}} \\
0 & 1 & 0 & 0
\end{array}\right), \quad \eta_{a b}:=\left(\begin{array}{cccc}
0 & 1 & 0 & 0 \\
1 & 0 & 0 & 0 \\
0 & 0 & 0 & 1 \\
0 & 0 & 1 & 0
\end{array}\right) .
$$

We now construct an ansatz for the deformed potentials $\Omega^{(n)}$ in terms of the deformed vierbein $e_{\mu}^{(n) a}$. Since we want that all the properties listed in Section 3.2 hold, we write first

$$
g_{\mu \nu}^{(n)}=\sum_{m=0}^{n} e_{\mu}^{(m) a} e_{\nu}^{(n-m) b} \eta_{a b}=\left(\delta_{\mu}^{i} \delta_{\nu}^{\bar{\jmath}}+\delta_{\nu}^{i} \delta_{\mu}^{\bar{\jmath}}\right) \Omega_{i \bar{\jmath}}^{(n)} .
$$

The simplest ansatz for the vierbein $e_{\mu}^{(n) a}$ that satisfies $g_{i j}^{(n)}=g_{\bar{\jmath} \mathfrak{( n ) *}}^{(n)}=0$, is

$$
e_{\mu}^{(n) a}=\left(\begin{array}{cccc}
0 & 0 & e_{\bar{p}}^{(n) 1} & e_{\bar{q}}^{(n) 1} \\
e_{p}^{(n) 2} & e_{q}^{(n) 2} & 0 & 0 \\
0 & 0 & e_{\bar{p}}^{(n) 3} & e_{\bar{q}}^{(n) 3} \\
e_{p}^{(n) 4} & e_{q}^{(n) 4} & 0 & 0
\end{array}\right)
$$

As we discussed previously, the vierbein $e_{\mu}^{(n) a}$ must have the property $\partial_{\mu} e_{\nu}^{(n) a}=0, \mu=p, q, \bar{p}, \bar{q}$. Therefore, we shall assume the following dependence

$$
e_{\mu}^{(n+1) a}=e_{\mu}^{(n+1) a}\left(\Omega_{p \bar{p}}^{(n)}, \Omega_{q \bar{q}}^{(n)}, \Omega_{p \bar{q}}^{(n)}, \Omega_{q \bar{p}}^{(n)}\right) .
$$


The deformed Kähler potential $\Omega^{(n+1)}$ to order $n+1$ will depend explicitly on the vierbein to order $n+1$ and $n$, i.e., on $e_{\mu}^{(n+1) a}$ and $e_{\mu}^{(n) a}$ respectively. We begin by analising the deformation to first order: from equation (5.2) we obtain

$$
\Omega_{i \bar{\jmath}}^{(1)}=e_{(i}^{(1) a} e_{\bar{\jmath})}^{b} \eta_{a b} .
$$

Using the undeformed vierbein $e_{\mu}^{a}$ given in equation (5.1), we write explicitly

$$
\begin{aligned}
& \Omega_{q \bar{q}}^{(1)}=e_{\bar{q}}^{(1) 3}+e_{q}^{(1) 2} \Omega_{p \bar{q}}+e_{q}^{(1) 4} \Omega_{q \bar{q}}, \\
& \Omega_{q \bar{p}}^{(1)}=e_{\bar{p}}^{(1) 3}+e_{q}^{(1) 2} \Omega_{p \bar{p}}+e_{q}^{(1) 4} \Omega_{q \bar{p}}, \\
& \Omega_{p \bar{p}}^{(1)}=e_{\bar{p}}^{(1) 1}+e_{p}^{(1) 2} \Omega_{p \bar{p}}+e_{p}^{(1) 4} \Omega_{q \bar{p}}, \\
& \Omega_{p \bar{q}}^{(1)}=e_{\bar{q}}^{(1) 1}+e_{p}^{(1) 2} \Omega_{p \bar{q}}+e_{p}^{(1) 4} \Omega_{q \bar{q}} .
\end{aligned}
$$

To obtain the Kähler potential up to first order, we need to solve equations (5.4)-(5.7). If we integrate first the above equations with respect to the anti-holomorphic variables $x^{\bar{\imath}}=\{\bar{p}, \bar{q}\}$, we need to calculate a set of integrals of the form $\int e_{\alpha}^{(1) A} \Omega_{\beta \mu} \mathrm{d} x^{\nu}$. After an integration by parts, we see that

$$
\int e_{\alpha}^{(1) A} \Omega_{\beta \mu} \mathrm{d} x^{\nu}= \begin{cases}e_{\alpha}^{(1) A} \Omega_{\beta \mu} x^{\nu}+C_{i}^{(1)}, & \text { if } \mu \neq \nu, \\ e_{\alpha}^{(1) A} \Omega_{\beta}+C_{i}^{(1)}, & \text { if } \mu=\nu,\end{cases}
$$

where we used the properties $\partial_{\mu} e_{\nu}^{(1) a}=0, \partial_{\alpha} \Omega_{\mu \nu}=0$ of the vierbein and the Kähler potential respectively. In consequence, we have

$$
\begin{aligned}
& \Omega_{q}^{(1)}=e_{\bar{q}}^{(1) 3} \bar{q}+e_{q}^{(1) 2} \Omega_{p}+e_{q}^{(1) 4} \Omega_{q}+C_{1}^{(1)}(p, q, \bar{p}), \\
& \Omega_{q}^{(1)}=e_{\bar{p}}^{(1) 3} \bar{p}+e_{q}^{(1) 2} \Omega_{p}+e_{q}^{(1) 4} \Omega_{q}+C_{2}^{(1)}(p, q, \bar{q}), \\
& \Omega_{p}^{(1)}=e_{\bar{p}}^{(1) 1} \bar{p}+e_{p}^{(1) 2} \Omega_{p}+e_{p}^{(1) 4} \Omega_{q}+C_{3}^{(1)}(p, q, \bar{q}), \\
& \Omega_{p}^{(1)}=e_{\bar{q}}^{(1) 1} \bar{q}+e_{p}^{(1) 2} \Omega_{p}+e_{p}^{(1) 4} \Omega_{q}+C_{4}^{(1)}(p, q, \bar{p}),
\end{aligned}
$$

where $C_{1}^{(1)}=C_{1}^{(1)}(p, q, \bar{p}), C_{2}^{(1)}=C_{2}^{(1)}(p, q, \bar{q}), C_{3}^{(1)}=C_{3}^{(1)}(p, q, \bar{q})$ and $C_{4}^{(1)}=C_{4}^{(1)}(p, q, \bar{p})$ are functions of their arguments. We determine these functions by comparing equations (5.8)-(5.9) and (5.10)-(5.11); we conclude that $C_{1}^{(1)}=e_{\bar{p}}^{(1) 3} \bar{p}, C_{2}^{(1)}=e_{\bar{q}}^{(1) 3} \bar{q}, C_{3}^{(1)}=e_{\bar{q}}^{(1) 1} \bar{q}$ and $C_{4}^{(1)}=e_{\bar{p}}^{(1) 1} \bar{p}$. Therefore, we obtain the following two expressions

$$
\begin{aligned}
& \Omega_{q}^{(1)}=e_{\bar{p}}^{(1) 3} \bar{p}+e_{\bar{q}}^{(1) 3} \bar{q}+e_{q}^{(1) 2} \Omega_{p}+e_{q}^{(1) 4} \Omega_{q}, \\
& \Omega_{p}^{(1)}=e_{\bar{p}}^{(1) 1} \bar{p}+e_{\bar{q}}^{(1) 1} \bar{q}+e_{p}^{(1) 2} \Omega_{p}+e_{p}^{(1) 4} \Omega_{q} .
\end{aligned}
$$

Following the same procedure as before, after an integration by parts of equations (5.12) and (5.13) with respect to the holomorphic variables $q$ and $p$ respectively, the Kähler potential takes the unique form

$$
\begin{aligned}
\Omega^{(1)}= & \left(e_{\bar{p}}^{(1) 1} p+e_{\bar{p}}^{(1) 3} q\right) \bar{p}+\left(e_{\bar{q}}^{(1) 1} p+e_{\bar{q}}^{(1) 3} q\right) \bar{q}+e_{q}^{(1) 2} \Omega_{p} q+e_{p}^{(1) 4} \Omega_{q} p \\
& +\left(e_{p}^{(1) 2}+e_{q}^{(1) 4}\right) \Omega .
\end{aligned}
$$

In general, a straightforward calculation shows that the Kähler potential up to the $n$-th order has the expression

$$
\begin{aligned}
\Omega^{(n)}= & \left(e_{\bar{p}}^{(n) 1} p+e_{\bar{p}}^{(n) 3} q\right) \bar{p}+\left(e_{\bar{q}}^{(n) 1} p+e_{\bar{q}}^{(n) 3} q\right) \bar{q}+e_{q}^{(n) 2} \Omega_{p} q+e_{p}^{(n) 4} \Omega_{q} p+\left(e_{p}^{(n) 2}+e_{q}^{(n) 4}\right) \Omega \\
& +\sum_{m=1}^{n-1}\left(e_{p}^{(m) a} p+e_{q}^{(m) a} q\right)\left(e_{\bar{p}}^{(n-m) b} \bar{p}+e_{\bar{q}}^{(n-m) b} \bar{q}\right) \eta_{a b} .
\end{aligned}
$$

When $n=1$, we recover equation (5.14). 


\subsection{Solutions for the Kähler potential to first order}

As a particular example of the previous approach, we consider now in detail the deformation of the Kähler potential and the vierbein up to first order on the noncommutative parameter $\theta$. We recall that the curvature condition can be formulated as a simple determinant, that is

$$
\operatorname{det} \widehat{\Omega}_{i \bar{\jmath}}=\widehat{\Omega}_{p \bar{p}} \widehat{\Omega}_{q \bar{q}}-\widehat{\Omega}_{p \bar{q}} \widehat{\Omega}_{q \bar{p}}=1,
$$

where $\widehat{\Omega}=\Omega+\theta \Omega^{(1)}$ up to first order. Substituting this expression in the determinant condition, we obtain the following equations

$$
\begin{aligned}
& \Omega_{p \bar{p}} \Omega_{q \bar{q}}-\Omega_{p \bar{q}} \Omega_{q \bar{p}}=1, \\
& \Omega_{p \bar{p}} \Omega_{q \bar{q}}^{(1)}+\Omega_{p \bar{p}}^{(1)} \Omega_{q \bar{q}}-\Omega_{p \bar{q}} \Omega_{q \bar{p}}^{(1)}-\Omega_{p \bar{q}}^{(1)} \Omega_{q \bar{p}}=0 .
\end{aligned}
$$

Equations (5.15) and (5.16) are the Monge-Ampère equations to zero and first order respectively. If we substitute now equations (5.4)-(5.7) into the heavenly equation to first order equation (5.16), we obtain

$$
e_{q}^{(1) 4}+e_{p}^{(1) 2}+e_{\bar{q}}^{(1) 3} \Omega_{p \bar{p}}+e_{\bar{p}}^{(1) 1} \Omega_{q \bar{q}}-e_{\bar{p}}^{(1) 3} \Omega_{p \bar{q}}-e_{\bar{q}}^{(1) 1} \Omega_{q \bar{p}}=0 .
$$

Therefore, we need to find a form for the vierbein $e_{\mu}^{(1) a}$ such that the previous equation holds. We consider the following two possibilities

$$
\Omega_{p \bar{p}}^{(1)}=C \Omega_{q \bar{p}}^{(1)}, \quad \Omega_{p \bar{q}}^{(1)}=C \Omega_{q \bar{q}}^{(1)},
$$

for case I, and

$$
\Omega_{p \bar{p}}^{(1)}=C \Omega_{p \bar{q}}^{(1)}, \quad \Omega_{q \bar{p}}^{(1)}=C \Omega_{q \bar{q}}^{(1)},
$$

for case II. We use now equations (5.4)-(5.7) into the previous formulas to obtain, after some simplifications, the following relations among the components of the vierbein to first order

$$
C e_{q}^{(1) 4}=e_{p}^{(1) 4}, \quad C e_{q}^{(1) 2}=e_{p}^{(1) 2}, \quad C e_{\bar{q}}^{(1) 3}=e_{\bar{q}}^{(1) 1}, \quad C e_{\bar{p}}^{(1) 3}=e_{\bar{p}}^{(1) 1},
$$

for case I, and

$$
\begin{aligned}
e_{\bar{p}}^{(1) 1} & =C e_{\bar{q}}^{(1) 1}, \quad e_{\bar{p}}^{(1) 3}=C e_{\bar{q}}^{(1) 3}, \quad e_{p}^{(1) 4}=\frac{A}{A^{\prime}} e_{q}^{(1) 4}=A\left(\Omega_{q \bar{p}}-C \Omega_{q \bar{q}}\right), \\
e_{p}^{(1) 2} & =\frac{A}{A^{\prime}} e_{q}^{(1) 2}=A\left(\Omega_{p \bar{p}}-C \Omega_{p \bar{q}}\right),
\end{aligned}
$$

for case II. $A, A^{\prime}$ and $C$ are arbitrary constants in the above expressions. After the respective simplifications, the Monge-Ampère equation becomes

$$
e_{q}^{(1) 4}+e_{p}^{(1) 2}+e_{\bar{q}}^{(1) 3}\left(\Omega_{p \bar{p}}-C \Omega_{q \bar{p}}\right)+e_{\bar{p}}^{(1) 1}\left(C \Omega_{q \bar{q}}-\Omega_{p \bar{q}}\right)=0,
$$

for case I and

$$
e_{q}^{(1) 4}+e_{p}^{(1) 2}+e_{\bar{q}}^{(1) 3}\left(\Omega_{p \bar{p}}-C \Omega_{p \bar{q}}\right)+e_{\bar{p}}^{(1) 1}\left(C \Omega_{q \bar{q}}-\Omega_{q \bar{p}}\right)=0,
$$

for case II.

To further proceed, we recall that according to equation (5.3) the vierbein $e_{\mu}^{(1) a}$ should have the functional dependence

$$
e_{\mu}^{(1) a}=e_{\mu}^{(1) a}\left(\Omega_{p \bar{p}}, \Omega_{q \bar{q}}, \Omega_{p \bar{q}}, \Omega_{q \bar{p}}\right),
$$


where $\Omega_{i \bar{\jmath}}$, with $\{i, \bar{\jmath}\}=\{p, q, \bar{p}, \bar{q}\}$, are the metric coefficients of the undeformed metric tensor. For case I, we choose the following ansatz for the vierbein

$$
\begin{aligned}
& e_{q}^{(1) 4}=\alpha+\beta \Omega_{p \bar{p}}+\gamma \Omega_{q \bar{q}}+\delta \Omega_{p \bar{q}}+\sigma \Omega_{q \bar{p}}, \\
& e_{p}^{(1) 2}=\alpha^{\prime}+\beta^{\prime} \Omega_{p \bar{p}}+\gamma^{\prime} \Omega_{q \bar{q}}+\delta^{\prime} \Omega_{p \bar{q}}+\sigma^{\prime} \Omega_{q \bar{p}}, \\
& e_{\bar{q}}^{(1) 3}=\alpha^{\prime \prime}+\beta^{\prime \prime} \Omega_{p \bar{p}}+\gamma^{\prime \prime} \Omega_{q \bar{q}}+\delta^{\prime \prime} \Omega_{p \bar{q}}+\sigma^{\prime \prime} \Omega_{q \bar{p}}, \\
& e_{\bar{p}}^{(1) 3}=\alpha^{\prime \prime \prime}+\beta^{\prime \prime \prime} \Omega_{p \bar{p}}+\gamma^{\prime \prime \prime} \Omega_{q \bar{q}}+\delta^{\prime \prime \prime} \Omega_{p \bar{q}}+\sigma^{\prime \prime \prime} \Omega_{q \bar{p}},
\end{aligned}
$$

where $\alpha, \alpha^{\prime}, \beta, \beta^{\prime}, \ldots$ are arbitrary constants. If we substitute the above equations into equation (5.17), we obtain the following relationship between the coefficients

$$
\begin{aligned}
& \alpha+\alpha^{\prime}+\gamma^{\prime \prime}+C \beta^{\prime \prime \prime}=0, \quad \beta+\beta^{\prime}+\alpha^{\prime \prime}=0, \quad \gamma+\gamma^{\prime}+C \alpha^{\prime \prime \prime}=0, \\
& \delta+\delta^{\prime}-\alpha^{\prime \prime \prime}=0, \quad \sigma+\sigma^{\prime}-C \alpha^{\prime \prime}=0 .
\end{aligned}
$$

The solution to this system of coupled linear equations is

$$
\begin{aligned}
& \gamma^{\prime \prime}=\sigma^{\prime \prime \prime}=-\delta^{\prime \prime}=-\beta^{\prime \prime \prime}, \quad \gamma^{\prime \prime \prime}=0=\delta^{\prime \prime \prime}, \quad \sigma^{\prime \prime}=0=\beta^{\prime \prime}, \\
& C=-\frac{\alpha+\alpha^{\prime}+\gamma+\gamma^{\prime}+\gamma^{\prime \prime}+\sigma+\sigma^{\prime}}{\beta^{\prime \prime \prime}-\alpha^{\prime \prime}+\alpha^{\prime \prime \prime}} .
\end{aligned}
$$

For case II, we choose the following ansatz for the vierbein

$$
\begin{aligned}
& e_{\bar{q}}^{(1) 1}=\alpha+\beta \Omega_{p \bar{p}}+\gamma \Omega_{q \bar{q}}+\delta \Omega_{p \bar{q}}+\sigma \Omega_{q \bar{p}}, \\
& e_{\bar{q}}^{(1) 3}=\alpha^{\prime}+\beta^{\prime} \Omega_{p \bar{p}}+\gamma^{\prime} \Omega_{q \bar{q}}+\delta^{\prime} \Omega_{p \bar{q}}+\sigma^{\prime} \Omega_{q \bar{p}},
\end{aligned}
$$

where $\alpha, \alpha^{\prime}, \beta, \beta^{\prime}, \ldots$ are arbitrary constants. Substitution of these equations into equation (5.18) leads to

$$
\begin{aligned}
& \gamma^{\prime}+C \beta=0, \quad \alpha^{\prime}+A^{\prime}=0, \quad \alpha-A=0, \\
& \delta-\gamma^{\prime}=0, \quad \sigma^{\prime}-\beta=0, \quad \delta+C \sigma^{\prime}=0,
\end{aligned}
$$

with solution

$$
\gamma=0=\sigma, \quad \delta^{\prime}=0=\beta^{\prime}, \quad C=-\frac{\gamma^{\prime}}{\sigma^{\prime}}=-\frac{\delta}{\beta} .
$$

The previous expressions determine the Kähler potential that is compatible with the Moyal deformation of the first heavenly equation. We have thus arrived to a multi-parameter family of solutions for the Moyal-deformed Kähler potential.

\section{Deformed $\eta$-self dual Riemman metric}

Following [9], we consider the algebraic description of four dimensional non-compact hyperKähler manifolds that possess at least one abelian isometry, i.e., a translational or rotational symmetry. For this purpose, we start by recalling the rotational character of the corresponding Killing vector fields. By its own definition, a Killing vector field $\xi_{\mu}$ satisfies $\nabla_{(\nu} \xi_{\mu)}=0$, while the self-duality of the anti-symmetric part $\nabla_{[\nu} \xi_{\mu]}$ provides the critical distinction between these two types of Killing vectors field $[9,16]: \xi_{\mu}$ is translational if it satisfies the condition

$$
\xi_{\alpha ; \beta}=\frac{1}{2} \eta \epsilon_{\alpha \beta}{ }^{\mu \nu} \xi_{\mu ; \nu}, \quad \text { with } \quad \eta= \pm 1 .
$$

Otherwise, we say that $\xi_{\mu}$ is rotational. 
On the other hand, let $g_{\alpha \beta}$ be a $\eta$-self dual Riemman 4-metric with Euclidean signature and let $\xi=\xi^{\alpha} \partial_{\alpha}=\partial / \partial \phi$ be a Killing vector of $g_{\alpha \beta}$. Then, locally we may write

$$
\mathrm{d} s^{2}=\frac{1}{V}\left(d \phi+\omega_{i} \mathrm{~d} x^{i}\right)^{2}+\gamma_{i j} \mathrm{~d} x^{i} d x^{j}
$$

with $V, \omega_{i}$ and $\gamma_{i j}$ being all independent of $\phi$; Greek indices run from 0 to 3 and Latin indices run from 1 to 3 .

In [28], a set of complex coordinates for the Atiyah-Hitchin (AH) metric was found as an alternative procedure to the twistor formalism $[6,24]$. In the analysis of magnetic monopoles interactions, the moduli space admits a metric formulation in the low-energy limit leading to the $\mathrm{AH}$ metric and scattering processes may be analysed in this way; the length element of the $\mathrm{AH}$ metric is [3]

$$
\mathrm{d} s^{2}=\beta^{2} \gamma^{2} \delta^{2} \frac{(\mathrm{d} k)^{2}}{\left(4 k^{2} k^{\prime 2} K^{2}\right)^{2}}+\beta^{2} \sigma_{x}^{2}+\gamma^{2} \sigma_{y}^{2}+\delta^{2} \sigma_{z}^{2},
$$

where

$$
\beta \gamma=-K^{2}\left(k^{\prime 2}+u\right), \quad \gamma \delta=K^{2}\left(k^{2}-u\right), \quad \beta \delta=-K^{2} u,
$$

and

$$
u=\frac{G(k)}{K(k)}, \quad G(k)=E(k)-k^{\prime 2} K(k), \quad k^{\prime 2}=1-k^{2} .
$$

The differential 1-forms $\sigma_{x}, \sigma_{y}$ and $\sigma_{z}$ in the metric are invariant under $\mathrm{SU}(2)[3,4]$; the Killing vector associated to the diagonal $\mathrm{U}(1)$ is $\xi=\partial / \partial \phi$. By casting the metric in the form equation (6.1), we establish the identifications

$$
\begin{aligned}
& \frac{1}{V}=\frac{1}{4}\left(\beta^{2} \sin ^{2} \theta \cos ^{2} \psi+\gamma^{2} \sin ^{2} \theta \sin ^{2} \psi+\delta^{2} \cos ^{2} \theta\right), \\
& \omega=\frac{1}{V}\left(\left(\gamma^{2}-\beta^{2}\right) \sin \psi \cos \psi \sin \theta \mathrm{d} \theta+\delta^{2} \cos \theta \mathrm{d} \psi\right),
\end{aligned}
$$

together with

$$
\begin{aligned}
& \gamma_{k^{2} k^{2}}=\frac{\beta^{2} \gamma^{2} \delta^{2}}{V\left(4 k^{2} k^{2} K^{2}\right)^{2}}, \quad \gamma_{\theta \theta}=\frac{1}{16}\left[\beta^{2} \gamma^{2} \sin ^{2} \theta+\delta^{2} \cos ^{2} \theta\left(\beta^{2} \sin ^{2} \psi+\gamma^{2} \cos ^{2} \psi\right)\right], \\
& \gamma_{\theta \psi}=-\frac{1}{16}\left[\delta^{2}\left(\gamma^{2}-\beta^{2}\right) \cos \theta \sin \theta \cos \psi \sin \psi\right] \\
& \gamma_{\psi \psi}=\frac{1}{16} \delta^{2} \sin ^{2} \theta\left(\beta^{2} \cos ^{2} \psi+\gamma^{2} \sin ^{2} \psi\right) .
\end{aligned}
$$

The corresponding Kähler potential has a nice simple form as a function of $\theta, \psi, k^{2}$, namely [28]

$$
\Omega=\frac{\beta \gamma+\gamma \delta+\delta \beta}{4}-J
$$

where

$$
J:=\frac{1}{8}\left[(\beta \gamma+\gamma \delta+\delta \beta)-\gamma \delta \sin ^{2} \theta \cos ^{2} \psi-\delta \beta \sin ^{2} \theta \sin ^{2} \psi-\beta \gamma \cos ^{2} \theta\right] .
$$

On the other hand, Boyer and Finley [9] studied the Killing vectors in self dual Euclidean Einstein spaces using the formalism of complex $\mathcal{H}$-spaces. They proved that it is always possible to choose complex coordinates such that either

$$
\xi=\partial_{p}+\partial_{\bar{p}} \quad \text { and } \quad \xi \Omega=0, \quad \text { or } \quad \xi=\mathrm{i}\left(p \partial_{p}-\bar{p} \partial_{\bar{p}}\right) \quad \text { and } \quad \xi \Omega=0,
$$


depending on whether the covariant derivative of the Killing vector $\xi$ is purely self-dual or not. In the previous formulae, $\Omega$ is a Kähler potential that satisfies the Monge-Ampère equation. Furthermore, they showed how to simplify the equation for $\Omega$ with the help of an appropriate Legendre transformation in both cases. For this purpose, they introduced a pair of complex coordinates $x^{i} \equiv(q, p)$ such that the second alternative in equation (6.3) is satisfied, and set

$$
p=\sqrt{r} \mathrm{e}^{\mathrm{i} \tilde{\theta}} .
$$

In consequence the Killing vector is $\xi=\partial / \partial \tilde{\theta}$ and

$$
\Omega \equiv \Omega(r, q, \bar{q})
$$

By definition of the Kähler potential, we have

$$
\mathrm{d} s^{2}=2 \Omega_{i \bar{\jmath}} \mathrm{d} x^{i} \mathrm{~d} x^{\bar{\jmath}}
$$

where $\Omega$ satisfies the Monge-Ampère or first heavenly equation

$$
\Omega_{p \bar{p}} \Omega_{q \bar{q}}-\Omega_{p \bar{q}} \Omega_{q \bar{p}}=1 .
$$

In terms of the variable $r$, the first heavenly equation (6.5) becomes

$$
\left(r \Omega_{r}\right)_{r} \Omega_{q \bar{q}}-r \Omega_{r q} \Omega_{r \bar{q}}=1 .
$$

Now, if $J:=r \Omega_{r}$ ( $J$ is conjugated to $\ln r$ with respect to $\Omega$ ), and use $(J, q, \bar{q})$ as a new choice of independent variables to rewrite equations (6.4) and (6.6), we obtain

$$
\Omega_{q \bar{q}}=r_{J}\left[\frac{r_{q} r_{\bar{q}}}{r\left(r_{J}\right)^{2}}+1\right]
$$

where we used $\Omega_{r q}=-r^{-1} r_{J}^{-1} r_{q}, \Omega_{r \bar{q}}=-r^{-1} r_{J}^{-1} r_{\bar{q}}$ and $J_{r}=r_{J}^{-1}$. Equation (6.4) becomes

$$
\mathrm{d} s^{2}=\frac{1}{2}\left\{\left(\frac{r}{r_{J}}\right)\left[2 \mathrm{~d} \tilde{\theta}+\frac{\mathrm{i}}{r}\left(r_{q} \mathrm{~d} q-r_{\bar{q}} \mathrm{~d} \bar{q}\right)\right]^{2}+\left(\frac{r_{J}}{r}\right)\left(\mathrm{d} J^{2}+4 r \mathrm{~d} q \mathrm{~d} \bar{q}\right)\right\},
$$

so that the line element $\mathrm{d} s^{2}$ has the same form as equation (6.1); this coordinate frame is referred as the Toda frame [6]. With $J$ given by equation (6.2), it follows that we have the identifications

$$
V=\frac{r_{J}}{2 r}, \quad \mathrm{~d} \phi+\omega_{i} \mathrm{~d} x^{i}=\mathrm{d} \tilde{\theta}+\frac{i}{2 r}\left(r_{q} \mathrm{~d} q-r_{\bar{q}} \mathrm{~d} \bar{q}\right), \quad \gamma_{i j} \mathrm{~d} x^{i} \mathrm{~d} x^{j}=\mathrm{d} J^{2}+4 r \mathrm{~d} q \mathrm{~d} \bar{q} .
$$

According to Sections 2 and 3, a Moyal deformed Kähler potential $\widehat{\Omega}$ could be given as a power series expansion on the noncommutative parameter. If we also impose the condition that the moduli space, which is the AH spacetime, preserves its (anti-)self-dual character under the deformation, we must demand that the rotational Killing symmetry be unchanged; this requirement happens if, and only if, each $\Omega^{(n)}$ in the series expansion of the deformed Kähler potential is a function only of $r, q, \bar{q}$. Therefore, under the assumption $\Omega^{(n)} \equiv \Omega^{(n)}(r, q, \bar{q})$, the original first heavenly equation for the modified Kähler potentials $\Omega^{(n)}$ becomes

$$
\sum_{m=0}^{s}\left\{\left(r \Omega_{r}^{(m)}\right) \Omega_{q \bar{q}}^{(s-m)}-r \Omega_{r q}^{(m)} \Omega_{r \bar{q}}^{(s-m)}\right\}=0, \quad s=1,2, \ldots
$$

Following the same procedure that for the undeformed case [28], we start by defining $J^{(n)}:=$ $r \Omega_{r}^{(n)}$ and we use $(J, q, \bar{q})$ as a new choice of independent variables to write $\Omega_{r q}^{(n)}=-r^{-1} J_{r}^{(n)} r_{q}$ 
and $\Omega_{r \bar{q}}^{(n)}=-r^{-1} J_{r}^{(n)} r_{\bar{q}}$. Using this result and after a lengthy calculation, we obtain the following iterative expression for $\Omega_{q \bar{q}}^{(n)}$

$$
\Omega_{q \bar{q}}^{(n)}=r_{J} J_{r}^{(n)}\left(\Omega_{q \bar{q}}^{(0)}-2 r_{J}\right)+\sum_{\substack{s+m=n \\ m \neq n, 0}} r_{J} J_{r}^{(s)}\left(J_{r}^{(m)} r^{-1} r_{q} r_{\bar{q}}-\Omega_{q \bar{q}}^{(m)}\right),
$$

where $n \geq 1$. The corresponding line element associated to the modified Kähler potential $\widehat{\Omega}$ is then

$$
\begin{aligned}
\mathrm{d} \widehat{S}^{2}= & \sum_{n=0}^{\infty} \theta^{n} J_{r}^{(n)} r_{J} \mathrm{~d} s^{2}+\left[\sum_{n=1}^{\infty}-\theta^{n} 2 r_{J} r_{J} J_{r}^{(n)}+\sum_{n=2}^{\infty} \theta^{n} \sum_{m=1}^{n-1} r_{J} J_{r}^{(n-m)}\right. \\
& \left.\times\left(J_{r}^{(m)} r^{-1} r_{q} r_{\bar{q}}-\Omega_{q \bar{q}}^{(m)}\right)\right] \mathrm{d} q \mathrm{~d} \bar{q} .
\end{aligned}
$$

It is important to stress that $\mathrm{d} \widehat{S}^{2}$ possesses the same symmetries as the line element $\mathrm{d} s^{2}$ of the undeformed Kähler potential, namely, they both share the same Killing vector $\xi=\partial / \partial \tilde{\theta}$. In terms of the coordinate pair $\left(\phi, x^{i}\right)$, we write equation (6.8) as

$$
\begin{aligned}
\mathrm{d} \widehat{S}^{2}= & \sum_{n=0}^{\infty} \theta^{n} J_{r}^{(n)} r_{J} \mathrm{~d} s^{2}+\sum_{n=0}^{\infty} \theta^{n} \sum_{m=1}^{n-1} r_{J} J_{r}^{(n-m)}\left(J_{r}^{(m)} r^{-1} r_{q} r_{\bar{q}}+\Omega_{q \bar{q}}^{(m)}\right) \\
& \times \frac{1}{4}\left[\gamma_{i j} \mathrm{~d} x^{i} \mathrm{~d} x^{j}-\mathrm{d} J^{2}\right] .
\end{aligned}
$$

In equation (6.9), each one of the contributions $J_{r}^{(n)}$ and $\Omega_{q \bar{q}}^{(n)}$ must be expressed in terms of $\phi=\tilde{\theta}$ and $x^{i}$. The procedure is straightforward, and we outline it up to first order on $\theta$ : first, since both the undeformed AH metric and its Kähler potential are known, the metric components $\Omega_{i \bar{j}}$ are calculated. Then, the first order corrections $\Omega^{(1)}$ and $e^{(1)}$ to the Kähler potential and the vierbien are obtained; from them the elements $J^{(1)}$ are also deduced. We obtain the final form by using the change of coordinates in equation (6.7).

\section{Conclusions}

We analysed the construction of a Moyal deformation of the first heavenly equation that preserves the integrability character of the corresponding Kähler potential, as it happens in the standard commutative scenario. For this purpose, we reviewed the Moyal deformation of the first heavenly equation, where the Moyal bracket replaces the standard Poisson bracket; accordingly, a modified potential replaces the commutative Kähler potential that satisfies the first heavenly equation. An expression for the modified potential as a series expansion on the noncommutative parameter exists, where each term in this expansion satisfies a partial differential equation [38].

In the standard commutative situation, the Kähler potential satisfying the first heavenly equation is integrable. We extended this property to the modified potential by demanding a set of conditions using the Moyal bracket; these conditions also helped to fix the form of the potential in such a way that it becomes Kähler.

We applied these results to the particular case of a Kähler potential associated to self-dual vacuum solutions to Einstein's equations, and we analysed the problem of determining each one of the contributions in the series expansion of the modified Kähler potential. We obtained then explicit expressions for the deformed vierbein up to first order on the noncommutative parameter. With this information, we obtained two multi-parameter solutions for the Kähler potential also to first order. 
Finally, we applied this approach to the calculation of the modified Kähler potential associated with the AH spacetime, also up to first order on the noncommutative parameter. By extending the procedure of constructing complex coordinates for the AH metric [28], we obtained thus the modified $\mathrm{AH}$ metric in terms of the standard commutative one and the noncommutative contributions to the Kähler potential. Taking into account that the AH metric describes the moduli space of interacting magnetic monopoles at low energies, our results aim to incorporate noncommutative effects on these interactions. Furthermore, since the reduction of the AH to the Taub-NUT metric gives the dynamics of two well-separated interacting monopoles at low energies in a classical context, we expect that a deformation induced by noncommutativity would be relevant for this dynamics as well.

It would be interesting also to apply our construction to other spaces, such as the EguchiHanson metric; due to its uncomplicated form, we may find a non-perturbative result for the deformed metric. Considering the unique properties of this metric as a gravitational instanton and its connection with orbifolds and D-branes in asymptotically locally Euclidean spaces, we may find interesting consequences in the context of string theory.

\section{Acknowledgements}

The authors would like to thank the referees for their valuable remarks and suggestions to improve this work. D. Martínez-Carbajal acknowledges support from Universidad Autónoma Metropolitana (UAM, México).

\section{References}

[1] Alexandrov S., Pioline B., Vandoren S., Self-dual Einstein spaces, heavenly metrics, and twistors, J. Math. Phys. 51 (2010), 073510, 31 pages, arXiv:0912.3406.

[2] Alvarez-Gaumé L., Freedman D.Z., Geometrical structure and ultraviolet finiteness in the supersymmetric $\sigma$-model, Comm. Math. Phys. 80 (1981), 443-451.

[3] Atiyah M.F., Hitchin N.J., Low energy scattering of nonabelian monopoles, Phys. Lett. A 107 (1985), 21-25.

[4] Atiyah M.F., Hitchin N.J., The geometry and dynamics of magnetic monopoles, M.B. Porter Lectures, Princeton University Press, Princeton, NJ, 1988.

[5] Atiyah M.F., Hitchin N.J., Singer I.M., Self-duality in four-dimensional Riemannian geometry, Proc. Roy. Soc. London Ser. A 362 (1978), 425-461.

[6] Bakas I., Remarks on the Atiyah-Hitchin metric, Fortschr. Phys. 48 (2000), 9-14, arXiv:hep-th/9903256.

[7] Bakas I., Sfetsos K., Toda fields of $\mathrm{SO}(3)$ hyper-Kähler metrics and free field realizations, Internat. J. Modern Phys. A 12 (1997), 2585-2611, arXiv:hep-th/9604003.

[8] Boyer C.P., The geometry of complex self-dual Einstein spaces, in Nonlinear Phenomena (Oaxtepec, 1982), Lecture Notes in Phys., Vol. 189, Springer, Berlin, 1983, 25-46.

[9] Boyer C.P., Finley III J.D., Killing vectors in self-dual, Euclidean Einstein spaces, J. Math. Phys. 23 (1982), $1126-1130$.

[10] Boyer C.P., Plebański J.F., An infinite hierarchy of conservation laws and nonlinear superposition principles for self-dual Einstein spaces, J. Math. Phys. 26 (1985), 229-234.

[11] Douglas M.R., Nekrasov N.A., Noncommutative field theory, Rev. Modern Phys. 73 (2001), 977-1029, arXiv:hep-th/0106048.

[12] Eguchi T., Gilkey P.B., Hanson A.J., Gravitation, gauge theories and differential geometry, Phys. Rep. 66 (1980), 213-393.

[13] Esposito G., From spinor geometry to complex general relativity, Int. J. Geom. Methods Mod. Phys. 2 (2005), 675-731, arXiv:hep-th/0504089.

[14] Finley III J.D., Plebański J.F., Further heavenly metrics and their symmetries, J. Math. Phys. 17 (1976), $585-596$. 
[15] García D.A., Plebański J.F., Seven parametric type- $D$ solutions of Einstein-Maxwell equations in the basic left-degenerate representation, Nuovo Cimento A 40 (1977), 224-234.

[16] Gegenberg J.D., Das A., Stationary Riemannian space-times with self-dual curvature, Gen. Relativity Gravitation 16 (1984), 817-829.

[17] Gibbons G.W., Hawking S.W., Classification of gravitational instanton symmetries, Comm. Math. Phys. 66 (1979), 291-310.

[18] Gibbons G.W., Manton N.S., Classical and quantum dynamics of BPS monopoles, Nuclear Phys. B 274 (1986), 183-224.

[19] Gibbons G.W., Olivier D., Ruback P.J., Valent G., Multicentre metrics and harmonic superspace, Nuclear Phys. B 296 (1988), 679-696.

[20] Gibbons G.W., Ruback P.J., The hidden symmetries of multi-centre metrics, Comm. Math. Phys. 115 (1988), 267-300.

[21] Hanany A., Pioline B., (Anti-)instantons and the Atiyah-Hitchin manifold, J. High Energy Phys. 2000 (2000), no. 7, 001, 23 pages, arXiv:hep-th/0005160.

[22] Hitchin N.J., Karlhede A., Lindström U., Roček M., Hyperkähler metrics and supersymmetry, Comm. Math. Phys. 108 (1987), 535-589.

[23] Husain V., Self-dual gravity as a two-dimensional theory and conservation laws, Classical Quantum Gravity 11 (1994), 927-937, arXiv:gr-qc/9310003.

[24] Ionas R.A., Elliptic constructions of hyperkähler metrics I: The Atiyah-Hitchin manifold, arXiv:0712.3598.

[25] Ko M., Ludvigsen M., Newman E.T., Tod K.P., The theory of $\mathcal{H}$-space, Phys. Rep. 71 (1981), 51-139.

[26] Moyal J.E., Quantum mechanics as a statistical theory, Proc. Cambridge Philos. Soc. 45 (1949), 99-124.

[27] Newman E.T., Porter J.R., Tod K.P., Twistor surfaces and right-flat spaces, Gen. Relativity Gravitation 9 (1978), 1129-1142.

[28] Olivier D., Complex coordinates and Kähler potential for the Atiyah-Hitchin metric, Gen. Relativity Gravitation 23 (1991), 1349-1362.

[29] Park Q.H., Self-dual gravity as a large- $N$ limit of the 2D non-linear sigma model, Phys. Lett. B 238 (1990), 287-290.

[30] Park Q.H., 2D sigma model approach to 4D instantons, Internat. J. Modern Phys. A 7 (1992), $1415-1447$.

[31] Penrose R., Nonlinear gravitons and curved twistor theory, Gen. Relativity Gravitation 7 (1976), 31-52.

[32] Penrose R., The nonlinear graviton, Gen. Relativity Gravitation 7 (1976), 171-176.

[33] Penrose R., Ward R.S., Twistors for flat and curved space-time, in General Relativity and Gravitation, Vol. 2, Plenum, New York - London, 1980, 283-328.

[34] Plebański J.F., Some solutions of complex Einstein equations, J. Math. Phys. 16 (1975), 2395-2402.

[35] Plebański J.F., Przanowski M., Rajca B., Tosiek J., The Moyal deformation of the second heavenly equation, Acta Phys. Polon. B 26 (1995), 889-902.

[36] Plebański J.F., Robinson I., Left-degenerate vacuum metrics, Phys. Rev. Lett. 37 (1976), 493-495.

[37] Plebański J.F., Torres del Castillo G.F., $\mathcal{H}$ spaces with an algebraically degenerate right side, J. Math. Phys. 23 (1982), 1349-1352.

[38] Strachan I.A.B., The Moyal algebra and integrable deformations of the self-dual Einstein equations, Phys. Lett. B 283 (1992), 63-66.

[39] Strachan I.A.B., Hierarchy of conservation laws for self-dual gravity, Classical Quantum Gravity 10 (1993), 1417-1423.

[40] Strachan I.A.B., The symmetry structure of the anti-self-dual Einstein hierarchy, J. Math. Phys. 36 (1995), 3566-3573, arXiv:hep-th/9410047.

[41] Strachan I.A.B., A geometry for multidimensional integrable systems, J. Geom. Phys. 21 (1997), 255-278, arXiv:hep-th/9604142.

[42] Szabo R.J., Quantum field theory on noncommutative spaces, Phys. Rep. 378 (2003), 207-299, arXiv:hepth/0109162.

[43] Takasaki K., Dressing operator approach to Moyal algebraic deformation of selfdual gravity, J. Geom. Phys. 14 (1994), 111-120, arXiv:hep-th/9212103. 